\title{
Potenciales Ventajas del Método Pseudoespectral en Auralizaciones
}

\author{
Potential Advantages of the Pseudospectral Method in Auralizations
}

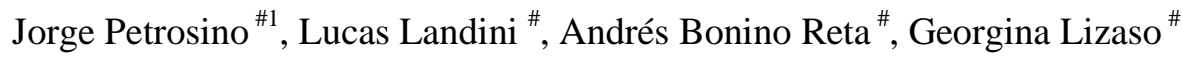 \\ * Departamento de Humanidades y Artes. Universidad Nacional de Lanús \\ 29 de Septiembre 3901, Remedios de Escalada, Buenos Aires, Argentina \\ 1 jorgepetrosino@gmail.com \\ Recibido: 08/12/18; Aceptado: 18/06/19
}

\begin{abstract}
Auralization is a term introduced to describe the recreation of the experience of acoustic phenomena a listener would perceive in a specific soundfield. Sound propagation in a soundfield can be simulated with geometric based models or wave based models. Each one offers particular advantages and disadvantages. For wave based models, the finite element method, the boundary element method or the finite difference method are widely mentioned. They are characterized for achieving very precise results for individual frequencies applied to small and moderately sized rooms. Geometric methods lead to the ray tracing method or the image source method. These methods achieve good results for high frequencies and are efficient in large rooms and complex structures, but are not able to represent in a simple manner specific wave phenomena such as diffraction. Commercial software used to produce auralizations is usually based on a hybrid model combining ray tracing and image sources. This paper proposes an exploration on possible advantages and challenges on the use of the k-space pseudospectral method for wave based auralizations.
\end{abstract}

Keywords: auralization; pseudospectral method; numerical methods

Resumen- El término auralización se refiere a la recreación en los oídos de un oyente de la sensación que percibiría en un espacio acústico determinado. La propagación del sonido en un espacio acústico puede simularse mediante procesos basados en el modelo geométrico o en el modelo ondulatorio. Cada uno ofrece ventajas $y$ dificultades particulares. Entre los modelos basados en ondas pueden mencionarse principalmente el método de elementos finitos, el de contornos finitos o el de diferencias finitas. Se caracterizan por lograr resultados muy precisos para frecuencias únicas aplicadas a recintos de tamaño pequeño o medio. Por otro lado, los modelos geométricos dan lugar al método del trazado de rayos o al método de las fuentes imagen. Estos métodos logran buenos resultados en frecuencias altas $y$ resultan eficientes en salas de gran tamaño con estructuras complejas, pero no pueden dar cuenta en forma sencilla de fenómenos específicamente ondulatorios como la difracción. Los programas comerciales utilizados para obtener auralizaciones suelen utilizar un modelo híbrido combinando el trazado de rayos y las fuentes imagen. El presente trabajo tiene la intención de iniciar una exploración sobre las posibles ventajas y desafíos del uso del método pseudoespectral del espacio $k$ para obtener auralizaciones basadas en el modelo ondulatorio.

Palabras clave: auralización; método pseudoespectral; métodos numéricos

\section{INTRODUCTION}

Vorländer define auralización como la técnica de crear señales en el rango audible mediante métodos numéricos, a partir de información simulada o medida que describa el espacio acústico, las fuentes y posición del oyente [1]. Los primeros intentos de auralización incluyeron modelos físicos a escala [2].

El proceso se basa en la determinación de la respuesta al impulso del espacio acústico a ser representado. Para ello se requiere seleccionar un punto en el que estará localizada la fuente en el espacio acústico. Dependiendo de la complejidad de la simulación, el modelo podría incluir patrones de directividad de la fuente sonora y de algunas otras características específicas, como la respuesta al impulso del sistema acústico o electroacústico que generará el sonido. Es necesario, además, disponer de un modelo matemático que represente el espacio acústico sobre el que se simulará la propagación del sonido, así como información sobre la ubicación del receptor y sus características.

La respuesta al impulso del modelo suele obtenerse mediante procesos basados en el modelo geométrico o en el modelo ondulatorio, cada uno de los cuales presenta ventajas y dificultades particulares.

Los modelos que se basan en acústica geométrica se implementan mediante el método del trazado de rayos (ray tracing method: RTM) o el método de las fuentes imagen (image source method: ISM). Algunos paquetes comerciales utilizan un método híbrido que combina la técnica de trazado de rayos con la de fuentes imagen [3]. Estos métodos logran buenos resultados en frecuencias altas y resultan eficientes en salas de gran tamaño con estructuras complicadas. Simulan la emisión de un impulso y sus reflexiones en las distintas superficies hasta alcanzar el punto de registro. La respuesta al impulso se obtiene en forma de un registro de esas múltiples reflexiones que forman lo que se conoce como ecograma. Estrictamente hablando no incluye todas las características esperables de una respuesta al impulso ya que el modelo geométrico no puede dar cuenta en forma sencilla de fenómenos específicamente ondulatorios como la difracción.

Entre los modelos basados en ondas pueden mencionarse principalmente el método de elementos finitos (finite element method: FEM) y el de contornos finitos (boundary 
element method: BEM). Estos se caracterizan por lograr resultados muy precisos para frecuencias únicas aplicadas a recintos de tamaño pequeño o medio [1]. Estos métodos resuelven las ecuaciones diferenciales convirtiendo las variables continuas en variables discretas, lo que provoca un efecto de dispersión numérica. Esto vuelve impráctico el estudio de casos en los que la fuente emite una onda de audio compleja (como la palabra hablada) o un impulso, y por ello quedan limitados a simulaciones para frecuencias únicas. Para poder obtener la respuesta al impulso se requiere un complejo trabajo de solución independiente para frecuencias puntuales o bandas estrechas de frecuencia cuyos resultados luego deben combinarse para conformar una respuesta al impulso global, lo que conlleva un aumento considerable del tiempo de cómputo.

El presente trabajo propone el uso de un método diferente de resolución de las ecuaciones diferenciales de propagación de ondas para generar auralizaciones. El método pseudoespectral del espacio k (k-space pseudospectral method) podría permitir realizar simulaciones tanto para ondas de audio complejas como para un impulso en una única sesión de cómputo, ubicándolo como una interesante alternativa a los métodos ondulatorios tradicionales

\section{A. Zonas de Validez de los Distintos Modelos de Comportamiento Acústico de Recintos}

A mediados del siglo XX, Manfred Schroeder analizó la región de transición de la respuesta en frecuencia de recintos que separa la región de baja frecuencia dominada por modos separados y la región de frecuencias dominada por una gran superposición de modos, en donde sería posible considerar ciertas variables de tipo estadístico.

Esta frecuencia de transición depende del tiempo de reverberación y del volumen del recinto. En 1954 [4], Schroeder propuso obtener el valor de dicha frecuencia como se muestra en la Ec. (1)

$$
\mathrm{fs}=\mathrm{K}_{\mathrm{s}} \cdot \sqrt{\frac{\mathrm{T}_{60}}{V_{\mathrm{ol}}}}
$$

En la publicación original, Schoeder utiliza un valor de $\mathrm{K}_{\mathrm{s}}=4000$. En 1962 [5], Schroeder y Kuttruff revisan dicha propuesta, reduciendo el factor a la mitad $K_{s}=2000$. Fuchs indica que es difícil considerar un valor inequívoco para este factor, por lo que propone considerar una franja indeterminada de frecuencias entre ambos valores de $\mathrm{Ks}$ citando diversos autores que argumentan respecto de la utilización de uno u otro valor [6].

Debido a la limitada capacidad de cálculo disponible en la época de la publicación original, la única manera de tratar con recintos de estructuras complejas era utilizando métodos estadísticos, lo que volvía imprescindible conocer los límites de validez de dichos métodos. La propuesta de Schroeder y Kuttruff permitía determinar una frecuencia límite. Los métodos estadísticos podían ofrecer resultados adecuados para frecuencias superiores a $f s$. Por debajo de esa frecuencia era necesario utilizar la teoría ondulatoria, que sólo podía resolverse para casos limitados al uso de formas geométricas sencillas. Por otra parte, el rango de frecuencias por sí solo no es garantía de validez de un modelo estadístico ya que se requiere incorporar otros criterios, como por ejemplo el de la distribución uniforme de materiales acústicos en el recinto.

La disponibilidad de computadoras hizo posible el trabajo con modelos basados en acústica geométrica, que asumen un comportamiento regido por reflexiones especulares para analizar el comportamiento de recintos. Para que los resultados obtenidos mediante estos modelos puedan considerarse adecuados es necesario que las longitudes de onda sean menores que el tamaño de las superficies sobre las que impactan. Esta condición impone restricciones importantes al rango de frecuencias ya que para tener en cuenta la influencia de irregularidades pequeñas en las superficies, el estudio queda limitado a valores muy elevados de frecuencias. La transición entre el comportamiento como onda y como rayo es gradual y resulta difícil de determinar con un único valor de frecuencia.

Es importante mencionar que tanto el modelo geométrico como el estadístico tienen una frecuencia mínima de validez, sin embargo los modelos ondulatorios no se encuentran limitados en frecuencia. Su alcance sólo podría verse comprometido por la capacidad de cómputo o por dificultades en la definición de modelos precisos de los elementos físicos incorporados al mismo.

La Fig. 1 muestra valores de fs para distintos tiempos de reverberación y volúmenes entre $100 \mathrm{~m}^{2}$ y $1200 \mathrm{~m}^{3}$.

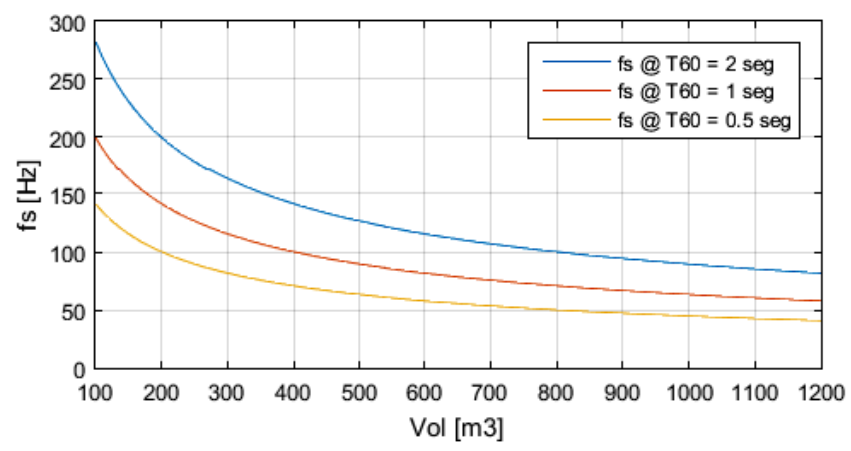

Fig. 1. Frecuencia de Schroeder (fs) para distintos tiempos de reverberación, considerando $\mathrm{Ks}=2000$.

\section{B. Modelos Basados en Acústica Geométrica}

Existen principalmente dos métodos que se basan en el modelo geométrico: el de trazado de rayos y el de las fuentes imagen.

El método del trazado de rayos genera un haz de rayos que parte de la fuente. La densidad de rayos utilizada en el análisis depende de la implementación particular, lo que puede dar lugar en casos poco densos a que terminen omitiéndose las reflexiones en alguna superficie de poca extensión. Cuando se detecta que un rayo interseca un elemento se genera una reflexión especular a partir de dicho punto. Los métodos de rayos no tienen en cuenta la longitud de onda específica, ni los valores instantáneos de presión o velocidad de partículas, sino que toman en consideración la energía transmitida. La reflexión en una determinada superficie puede ser afectada por un coeficiente de absorción. En casos más detallados es posible simular superficies difusas, considerando que en el punto de intersección existe una nueva fuente de energía sonora 
emitiendo en todas direcciones según la ley del coseno de Lambert. Este método no puede dar cuenta de los fenómenos puramente ondulatorios, como interferencia o difracción, aunque existen modos de incorporar procesos complementarios para suplir esta falencia. La cantidad de variantes propuestas es demasiado grande para dar cuenta aquí de todas las alternativas. Como ejemplo de algunas de estas variantes, podemos mencionar que existen versiones que utilizan procesos estocásticos para emular la absorción, haciendo que algunos rayos o partículas incidentes se reflejen y otros sean eliminados.

El método de las fuentes imagen reemplaza cada superficie reflectante por una fuente virtual a distancia, como si se tratase del reflejo en un espejo plano. En principio esto permite concentrar la capacidad de cómputo solamente en aquellos rayos que unen a las fuentes imagen con el punto de registro. Las fuentes creadas por la primera reflexión constituyen las fuentes imagen de primer orden, resultando necesario utilizar un orden tan alto como cantidad de reflexiones deseen simularse. Los coeficientes de absorción pueden tenerse en cuenta atenuando la intensidad de las fuentes imagen. Cuanto más tiempo de simulación se requiera, más fuentes imagen de orden superior será necesario incorporar. La principal diferencia en eficiencia con el trazado de rayos es que requiere menos capacidad de cómputo para tiempos cortos, como los correspondientes a fuentes imagen de órdenes bajos, pero crece en forma exponencial superando al requerido por el método de rayos al simular tiempos mayores.

El método de fuentes imagen no resulta adecuado para trabajar con superficies curvas, para incluir reflexión difusa, ni trabajar con espacios de formas complejas que den lugar a fuentes imagen ocultas, como por ejemplo al considerar recintos acoplados. La difracción no puede ser representada mediante este método. Podría sin embargo incorporarse información de fase para simular interferencia. Esta aparente ventaja no termina de ser suficientemente valiosa, ya que los efectos de interferencia tienen mayor influencia en la zona de bajas frecuencias, donde no se cumplen los postulados del modelo geométrico.

El método de las fuentes imagen tiene mejor resolución temporal, sin embargo el de trazado de rayos posibilita la inclusión del fenómeno de difusión. Es por esto que un método híbrido, como adecuada combinación de ambos, puede proporcionar mejores aproximaciones a la respuesta al impulso de un recinto real. En general los programas de simulación acústica comerciales actuales utilizan las fuentes imagen para determinar las reflexiones tempranas y el trazado de rayos para las tardías, siendo un tema de investigación científica actual el determinar el tiempo adecuado de transición entre ambos [1].

Para poder tener en cuenta características que dependan de la frecuencia (como la absorción o la difusión) el trazado de rayos debe repetirse para cada una de las bandas de frecuencia determinadas en el análisis. Cada banda de frecuencia da lugar a un reflectograma (o ecograma) que posteriormente debe combinarse con el resto para obtener la respuesta al impulso del recinto. Esta respuesta al impulso luego se convoluciona con un archivo de audio para generar una señal representativa de aquello que escucharía un oyente ubicado en el lugar seleccionado.

\section{Modelos Basados en Acústica Ondulatoria}

Entre los modelos ondulatorios comúnmente utilizados pueden citarse el método de elementos finitos (FEM) y el de contornos finitos (BEM). Estos modelos permiten en principio tener en cuenta todos los fenómenos ondulatorios, por lo que no tienen limitación en cuanto a la utilización de superficies curvas, ni a la simulación de efectos de difracción, difusión o interferencia. Los elementos presentes en el recinto no se representan mediante coeficientes como los de absorción o difusión, sino en términos de sus propiedades físicas como la densidad, la velocidad de propagación del sonido interna, o el módulo de compresibilidad.

Con estos métodos se obtienen resultados muy precisos pero a un alto costo computacional. Uno de los inconvenientes de los métodos numéricos de solución de las ecuaciones diferenciales es que el tipo de procesamiento realizado genera dispersión numérica en las soluciones [7]. Esto provoca que la velocidad de propagación varíe con la frecuencia, lo que obliga a realizar simulaciones para frecuencias puntuales y luego superponer los resultados para obtener la respuesta al impulso final del recinto.

Tanto en el método de elementos finitos, como en el de contornos finitos el espacio acústico se divide en pequeños elementos de tamaño adecuado a las necesidades de la simulación. Para cada elemento se establecen relaciones entre las presiones y los desplazamientos (a través de ecuaciones diferenciales en derivadas parciales), que luego dan lugar en conjunto a la solución global aproximada.

Ambos reemplazan la tarea de resolver ecuaciones complejas sobre volúmenes o superficies complejas, por la de resolver una gran cantidad de ecuaciones simples sobre una gran cantidad de elementos simples.

La diferencia relativa entre ambos es que el método de elementos finitos resuelve la ecuación de onda en todo el espacio, mientras que el de contornos finitos resuelve las ecuaciones en el contorno que delimita el espacio de interés lo que significa menos elementos de cálculo. Sin embargo, esta aparente ventaja relativa es opacada en cuanto a su eficiencia computacional porque las matrices necesarias para elementos finitos son poco densas y simétricas, mientras que las de contornos finitos son densas y asimétricas [8].

Por otra parte, al resolver la ecuación de onda en todo el espacio, el tiempo de procesamiento requerido en elementos finitos no varía con la cantidad de puntos de registro, por lo que pueden obtenerse resultados para diferentes lugares del recinto en forma conjunta.

Estos métodos se utilizan actualmente para el cálculo en recintos de tamaño pequeño o mediano y para frecuencias bajas [9]. La referencia a recintos pequeños o medianos se hace porque es allí donde las frecuencias de Schroeder son relativamente elevadas como muestra la figura $1 \mathrm{y}$ en ellos los métodos geométricos no resultan satisfactorios. Para recintos de mayor tamaño la frecuencia de Schroeder es razonablemente baja y el problema podría resolverse con los métodos geométricos que son más eficientes.

Es importante prestar atención a que tanto los métodos geométricos como los ondulatorios mencionados anteriormente requieren, por motivos diferentes, realizar procesos de simulación considerando frecuencias únicas o bandas estrechas de frecuencias. Que luego componen los 
resultados que dan lugar a la respuesta al impulso. Desde un punto de vista computacional, un posible método que permitiese obtener la respuesta al impulso en forma completa durante un único proceso de simulación podría presentarse como una interesante alternativa, aún cuando el método en sí mismo no ofreciera particulares ventajas desde los requerimientos generales de cálculo.

\section{MÉtodo Pseudoespectral DEL EsPACIO K}

Los métodos espectrales son una clase de técnicas numéricas que resuelven ecuaciones diferenciales. La idea central es la de obtener la solución como suma de ciertas funciones básicas (sinusoidales, por ejemplo) y luego calcular los coeficientes que mejor satisfagan la ecuación diferencial [10].

Tienen relación cercana con los métodos de elementos finitos. La principal diferencia es que los métodos espectrales utilizan funciones básicas que son no nulas en todo el dominio, mientras que los elementos finitos utilizan funciones que son no nulas en pequeños subdominios. Podría decirse que los métodos espectrales adoptan un enfoque global mientras que los de elementos finitos utilizan un enfoque local [11] [12].

Los métodos pseudoespectrales son una subclase de los métodos espectrales. La diferencia principal es que han sido adaptados para representar las funciones en una grilla rectangular, lo que simplifica la evaluación de ciertos operadores y puede incrementar la velocidad de cálculo permitiendo el uso de la transformada rápida de Fourier. El nivel de precisión de los métodos espectrales y los pseudoespectrales es muy similar [13] [14].

Cuando una onda acústica pasa a través de un medio se producen variaciones en la presión, la densidad, la temperatura, la velocidad de las partículas y otras variables. Estos cambios pueden describirse mediante ecuaciones diferenciales de primer orden acopladas que tienen en cuenta la conservación del momento, de la masa y de la energía en el medio. Es común en acústica combinar estas ecuaciones en relación con una variable (que puede ser la presión sonora o la velocidad de las partículas) formando una ecuación diferencial de segundo orden.

El método pseudoespectral del espacio $\mathrm{k}$ aplicado a la propagación de ondas acústicas resuelve las ecuaciones diferenciales de primer orden, en lugar de utilizar la ecuación combinada de segundo orden [15]. Esto se hace por dos motivos. En primer lugar, porque permite incluir de manera sencilla fuentes de masa y de fuerza en las ecuaciones discretas. En segundo lugar, porque habilita a utilizar una capa especial anisotrópica conocida como capa perfectamente acoplada (PML: perfectly matched layer) con el fin de absorber las ondas acústicas que alcanzan los bordes del dominio utilizado para el cálculo [16].

El método pseudoespectral del espacio $\mathrm{k}$ ha sido utilizado profusamente en los últimos años en aplicaciones biomédicas relacionadas con la propagación de ondas ultrasónicas en tejidos [17] [18]. También ha sido utilizado para el estudio de la alteración producida por variaciones de la temperatura atmosférica en la propagación de ondas mecánicas [19]. Hemos hallado muy pocos trabajos que utilicen métodos espectrales en acústica de recintos [20].

Hace poco menos de una década Treeby, Cox (University College London) y Jaros (Brno University of Technology) desarrollaron un paquete complementario (toolbox) para MATLAB u Octave que implementa de modo eficiente el método subespectral del espacio $\mathrm{k}$. El paquete fue diseñado para permitir simulaciones en el dominio del tiempo de ondas acústicas propagándose en medios complejos. Si bien su uso puede ser más general, fue diseñado especialmente para el estudio de la propagación de ondas ultrasónicas en tejido biológico en relación con imaginería médica [21].

Nuestro equipo viene trabajando con el paquete k-wave en diversas aplicaciones acústicas. Resulta muy sencilla la incorporación de un número ilimitado de fuentes sonoras con comportamientos temporales que pueden ser arbitrariamente definidos por el usuario utilizando cualquier tipo de función de MATLAB/Octave. Permite realizar simulaciones en una, dos o tres dimensiones. Pueden combinarse fuentes de presión y de velocidad. También pueden incorporarse una cantidad arbitraria de puntos de registro de presión y/o velocidad, sin límite y sin que esto implique mayor tiempo computacional, ya que el método resuelve siempre las ecuaciones en todos los puntos de su grilla rectangular. Por otra parte, pueden especificarse valores de densidad, velocidad de propagación y coeficiente de atenuación en cada punto de la grilla en forma independiente.

Los métodos numéricos tradicionales basados en el modelo ondulatorio producen errores en la estimación del gradiente, lo que da lugar a una dependencia de la velocidad del sonido con la frecuencia, generando una clase de dispersión numérica sin sentido físico. Los métodos pseudoespectrales reemplazan el cálculo de los gradientes espaciales mediante diferencias finitas por el cálculo espectral del gradiente por medio de series de Fourier. Esto evita la dispersión debida a la discretización espacial pero no aquella que surge de la aproximación de gradientes temporales por diferencias finitas. Sin embargo, el error cometido por el salto discreto temporal puede corregirse para un medio homogéneo hasta el límite de Nyquist [22].

Estas características resultan suficientemente interesantes como para iniciar un análisis exploratorio sobre la potencialidad de su utilización en auralizaciones intentando obtener la respuesta al impulso en un solo ciclo de simulación.

\section{PROPAGACIÓN DE UN IMPULSO EN 3D}

Los métodos clásicos que implementan el modelo ondulatorio trabajan simulando el comportamiento ante frecuencias puras o bandas estrechas para luego componer los resultados en una única respuesta al impulso general. Esto implica generalmente repetir el proceso innumerables veces, como queda claramente expresado por Aretz y cols. [23] al indicar que: "los resultados fueron calculados para el rango de frecuencias entre $200 \mathrm{~Hz}$ y $2500 \mathrm{~Hz}$ con saltos de $1 \mathrm{~Hz}$ ". Asumiendo un tiempo de cómputo de 5 minutos por frecuencia [1] esto tomaría más de 190 horas continuas de cómputo.

En esta sección se explora la posibilidad de obtener la respuesta al impulso en forma directa simulando la propagación en un espacio de tres dimensiones de una fuente impulsiva en una sola sesión de cómputo. La hipótesis de trabajo es que la simulación de la propagación de un impulso en un espacio de tres dimensiones desde la fuente hasta un sensor ubicado a cierta distancia provocará 
un registro que mantendrá las características del impulso original, con poca o nula deformación.

Tanto en los métodos pseudoespectrales como en los de elementos finitos se analiza la estabilidad de las soluciones mediante el coeficiente descripto en la Ec. (2) conocido como CFL (Courant - Friedrichs - Lewy). Corresponde a la razón entre la distancia que una onda avanzará en un salto temporal elemental y el salto de grilla.

$$
\mathrm{CFL}=\operatorname{cmax} \cdot \frac{\Delta \mathrm{t}}{\Delta \mathrm{x}}
$$

Los valores particulares de CFL que corresponden a condiciones de estabilidad cambian cuando se comparan modelos numéricos diferentes, como los pseudoespectrales y los de elementos finitos. Por ejemplo, un CFL $=0.3$ con 2 puntos por longitud de onda resultará en un salto temporal $\Delta \mathrm{t}$ cinco veces mayor al que corresponde a un modelo de diferencias finitas con 10 puntos por longitud de onda y el mismo valor de CFL [24] [25]. En las simulaciones se utilizó un valor $\mathrm{CFL}=0.3$ por presentar un adecuado balance entre precisión y velocidad de cómputo para medios homogéneos o ligeramente heterogéneos. El valor de salto temporal que utiliza internamente el k-wave se obtiene mediante la Ec. (3)

$$
\Delta \mathrm{t}=\frac{\mathrm{CFL} \cdot \Delta \mathrm{x}}{\mathrm{cmax}}
$$

Considerando valores de $\mathrm{CFL}=0.3, \Delta \mathrm{x}=1 \mathrm{~cm}, \mathrm{cmax}=$ $343 \mathrm{~m} / \mathrm{s}$, obtenemos un $\Delta \mathrm{t}=8.75 \mu \mathrm{s}$. Este $\Delta \mathrm{t}$ no se corresponde con un período de muestreo en el sentido utilizado normalmente en procesamiento de audio. La frecuencia máxima de validez para las funciones temporales que corresponden a las fuentes o a los registros se obtiene mediante la Ec. (4), donde cmin es la velocidad mínima de propagación en el medio.

$$
\mathrm{f}_{\max }=\frac{\mathrm{cmin}}{2 . \Delta \mathrm{x}}
$$

Se llevó a cabo la simulación de la propagación de una señal impulsiva en un espacio tridimensional de $256 \times 256 \times 256$ puntos de grilla, lo que da un total de más de 16 millones de nodos, representando un espacio cúbico de 2,56 m de lado con un salto discreto de $1 \mathrm{~cm}$ para cada una de las dimensiones espaciales. La frecuencia máxima de validez obtenida mediante la Ec. (4) fue de $17150 \mathrm{~Hz}$. La fuente se ubicó en las coordenadas ( $0 \mathrm{~m} ;-1 \mathrm{~m} ; 0 \mathrm{~m})$, con el sensor ubicado en $(0.5 \mathrm{~m} ; 0.5 \mathrm{~m} ; 0.5 \mathrm{~m})$. Esto da una distancia entre la fuente y el sensor de $1.66 \mathrm{~m}$. El tiempo de cómputo en una computadora personal (procesador i7 de 3.4 $\mathrm{GHz}$ con $16 \mathrm{GHz}$ de RAM) fue de $32 \mathrm{~min}$. La Fig. 2 muestra el espectro del registro del sensor. El caso ideal implicaría que el registro tuviese un espectro de módulo constante para todas las frecuencias. Los cálculos previos predicen una frecuencia máxima válida para la simulación de $17150 \mathrm{~Hz}$. Puede observarse en nuestros resultados que si se considera aceptable una variación menor a $1 \mathrm{~dB}$ en la respuesta al impulso, el rango de frecuencia debería limitarse en este caso a $14 \mathrm{kHz}$.

Estos resultados son perfectamente compatibles con la hipótesis de que es posible obtener la respuesta al impulso completa en un sólo proceso de simulación.

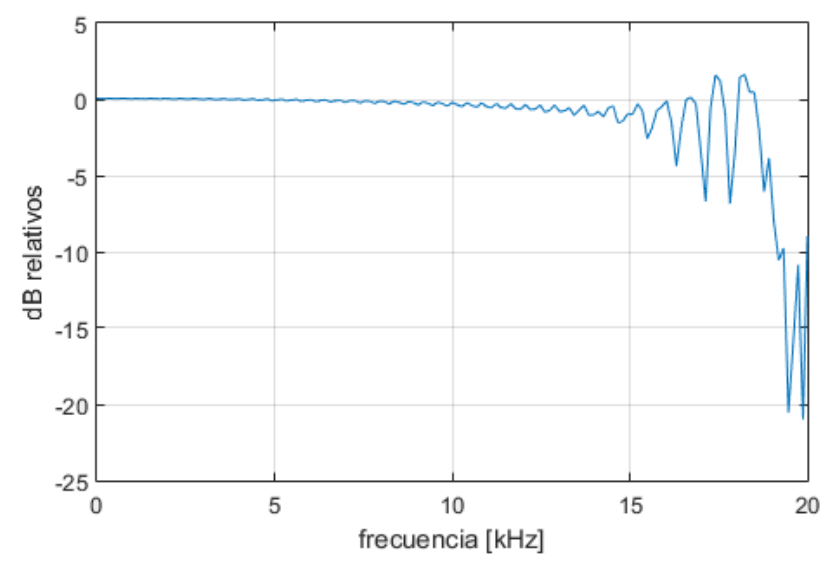

Fig. 2. Espectro de la respuesta al impulso (módulo de la función de transferencia del sistema)

El tiempo de cómputo reportado en esta sección sólo tuvo en cuenta la propagación desde la fuente hasta el sensor, con el fin de poner a prueba la hipótesis de que el impulso no se altera al propagarse, a diferencia de lo que sucede en los métodos de elementos finitos. Dicho tiempo de cómputo aumentará si se intenta obtener auralizaciones en un recinto para poder registrar las sucesivas reflexiones. De todas maneras, estos tiempos resultan inferiores que los esperables por la repetición de simulaciones mediante elementos finitos para cada frecuencia por separado.

Es importante insistir aquí que dado que se resuelven las ecuaciones diferenciales en todo el espacio, el tiempo de cómputo no depende en forma directa de la cantidad de sensores incorporados a la simulación, por lo cual pueden utilizarse gran cantidad de puntos de registro, obteniendo en la misma sesión de cómputo múltiples auralizaciones.

\section{IV.PROPAGACIÓN DE ONDAS COMPLEJAS EN 2D}

Como se mencionó anteriormente, el proceso de auralización consiste en obtener la respuesta al impulso en un recinto para convolucionar luego esta respuesta al impulso con una señal de audio. Este proceso genera un audio final con las características de lo que sería registrado por un oyente ubicado en donde se encuentra el sensor en la simulación.

Claramente simular solamente la propagación de un impulso supone una ventaja en tiempo de cómputo respecto de lo que implicaría simular la propagación de todo el audio ya que el registro de la respuesta al impuso sólo requiere simular el tiempo necesario para que las reflexiones disminuyan hasta un nivel considerado suficiente para la simulación. Este tiempo de simulación es independiente de la duración del audio. Se asume que la convolución de la respuesta al impuso con el archivo de audio sin procesar genera un resultado similar a la que provocaría el audio si fuese emitido desde la fuente. Para que esto sea válido es necesario que el sistema sea lineal e independiente en el tiempo (sistema LTI, por linear time invariant). La entrada al sistema es la señal emitida por la fuente, la salida es el registro y el sistema es todo el espacio por el cual las ondas se propagan.

Con el método pseudoespectral del espacio $\mathrm{k}$ fue posible realizar una prueba de concepto para evaluar la linealidad del sistema al considerar la propagación de ondas. Esta prueba consistió en obtener una auralización de la 
propagación de todo el audio completo correspondiente a un registro de la palabra "hola" y comparar este resultado con la obtención de una respuesta al impulso y su posterior convolución con el registro de la palabra original.

La simulación de la propagación de un audio complejo no resulta posible con los métodos tradicionales, pero puede en cambio desarrollarse con el método pseudoespectral. El tiempo de cómputo para simular la propagación de un audio completo es muy elevado, por lo cual se decidió para esta prueba de concepto utilizar una versión limitada a dos dimensiones, lo que disminuye enormemente la cantidad de nodos a considerar.

\section{A. Propagación Directa de un Archivo de Audio}

En la simulación realizada se utilizó una única velocidad de propagación en medio homogéneo (aire), con lo cual $\mathrm{cmin}=\mathrm{cmax}=343 \mathrm{~m} / \mathrm{s}$. Con los valores seleccionados se obtuvo una frecuencia fmax $=17150 \mathrm{~Hz}$

Se definió un espacio de propagación de 2,56 m de lado. Estos valores fueron elegidos para dar lugar a una grilla cuadrada de 256 puntos de lado (65536 puntos en la grilla) que resulta computacionalmente eficiente para el método pseudoespectral. El toolbox k-wave dispone de una función denominada checkFactors() que facilita tomar decisiones respecto de valores de grilla eficientes para el cálculo.

Se colocó una fuente en $\mathrm{x} 0=0 \mathrm{~m} ; \mathrm{y} 0=-1 \mathrm{~m}$ y un sensor de presión sonora en $\mathrm{x} 0=0 \mathrm{~m} ; \mathrm{y} 0=1 \mathrm{~m}$. Se impuso a la fuente de presión la condición de ser tipo Dirichlet (obligando de este modo a que el valor de presión en el punto correspondiente a la fuente sea exactamente el especificado). En esta experiencia se pretendía analizar solamente el comportamiento de propagación por lo que el sonido se propaga en el aire sin obstáculos ni paredes

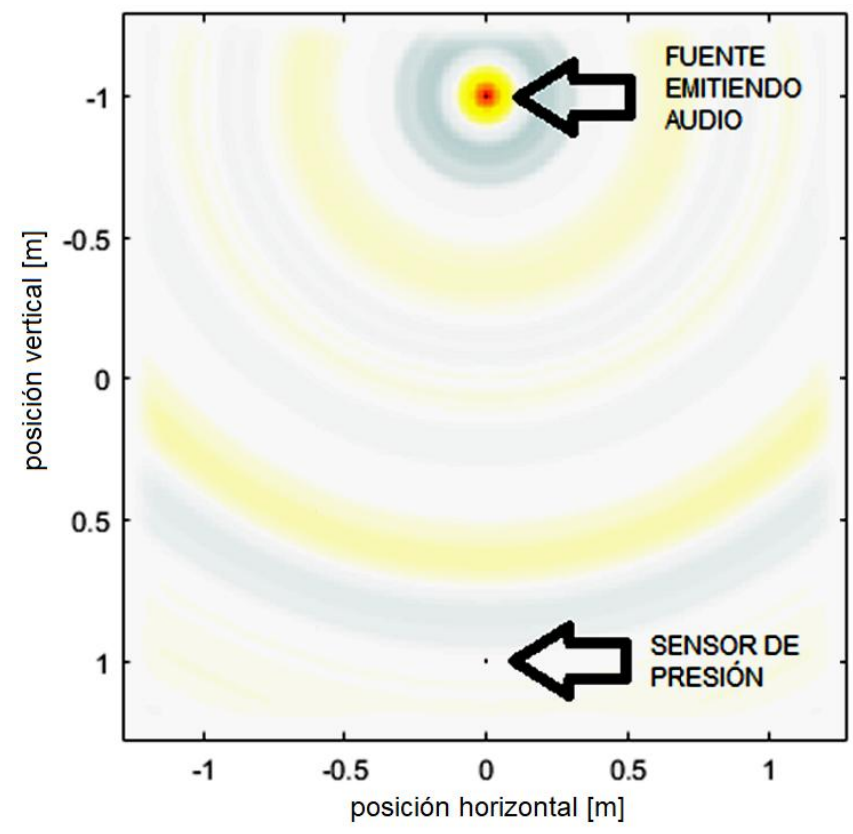

Fig. 3. Representación espacial de un instante en la propagación de una señal de audio a lo largo del espacio definido en la simulación

El desarrollo temporal de la fuente fue especificado a partir de la información registrada previamente en un archivo de audio de palabra hablada. Se registró una palabra breve (hola) cuya duración temporal fue de $317 \mathrm{~ms}$. El audio fue registrado con una frecuencia de muestreo de 48 $\mathrm{kHz}$.

Dado que k-wave opera con un $\Delta \mathrm{t}$ muy inferior al del período de muestreo, fue necesario remuestrear el audio para que se adapte al $\Delta$ t utilizado en la solución de las ecuaciones diferenciales de propagación. Se aplicó la función resample() para llevar la señal de audio original muestreada a $48 \mathrm{kHz}$ a una señal muestreada a $114 \mathrm{kHz}$.

Este audio remuestreado se constituyó en la información de desarrollo temporal de la fuente de presión tipo Dirichlet. La propagación de toda la información de audio a lo largo de los 2 metros que separaban la fuente del sensor tomó un tiempo de cálculo de 4 min en una computadora personal de escritorio (procesador i7 de $3.4 \mathrm{GHz}$ con $16 \mathrm{GHz}$ de RAM). El toolbox ofrece al comienzo de la simulación una estimación del tiempo de cómputo total que requerirá la simulación. La Fig. 3 muestra un instante de la simulación de propagación del audio.

El audio registrado por el sensor fue reproducido directamente en MATLAB con una frecuencia de muestreo de $114 \mathrm{kHz}$. Resultó perfectamente claro, con un ligero incremento de frecuencias bajas.
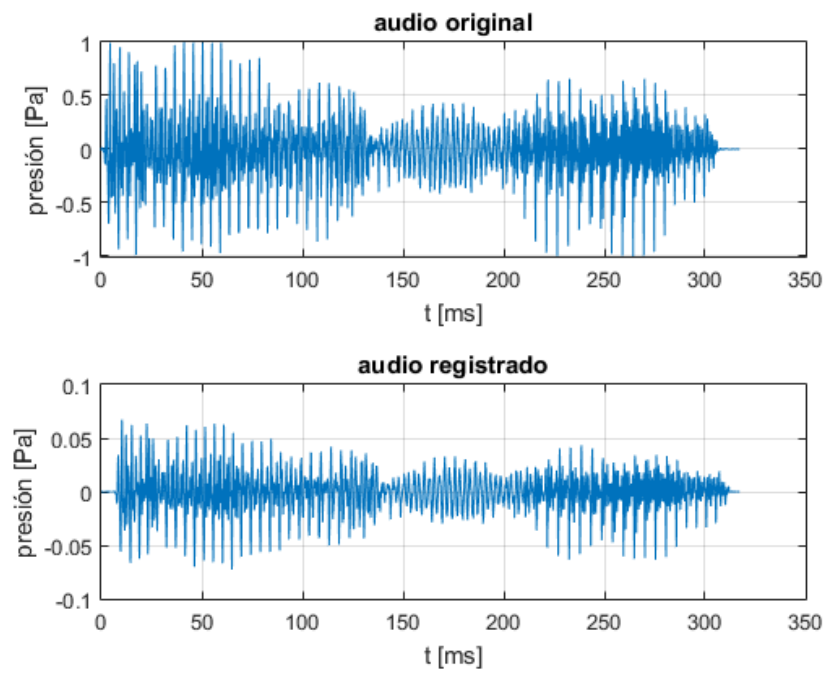

Fig. 4. Diagramas temporales del audio original emitido por la fuente y el audio registrado por el sensor.

La Fig. 4 muestra los diagramas temporales del audio emitido por la fuente y el audio captado por el sensor ubicado a $2 \mathrm{~m}$ de distancia. Puede notarse un breve retardo en el registro correspondiente al tiempo de propagación entre la fuente y el sensor.

\section{B. Propagación de un Impulso}

Para llevar a cabo la segunda simulación se mantuvo la configuración anterior utilizando una fuente impulsiva. Se eligió un tiempo total de procesamiento ligeramente superior al de propagación del impulso ( $\mathrm{t}$ ffinal $=6.3 \mathrm{~ms}$ ). En este caso el tiempo total de cómputo fue de sólo 6 segundos. La Fig. 5 muestra el diagrama temporal y el espectral de la respuesta al impulso. El incremento en frecuencias bajas que se observa en la respuesta en frecuencia es producto de la utilización de una fuente puntual de tipo Dirichlet cuando se realiza una simulación en dos dimensiones. 

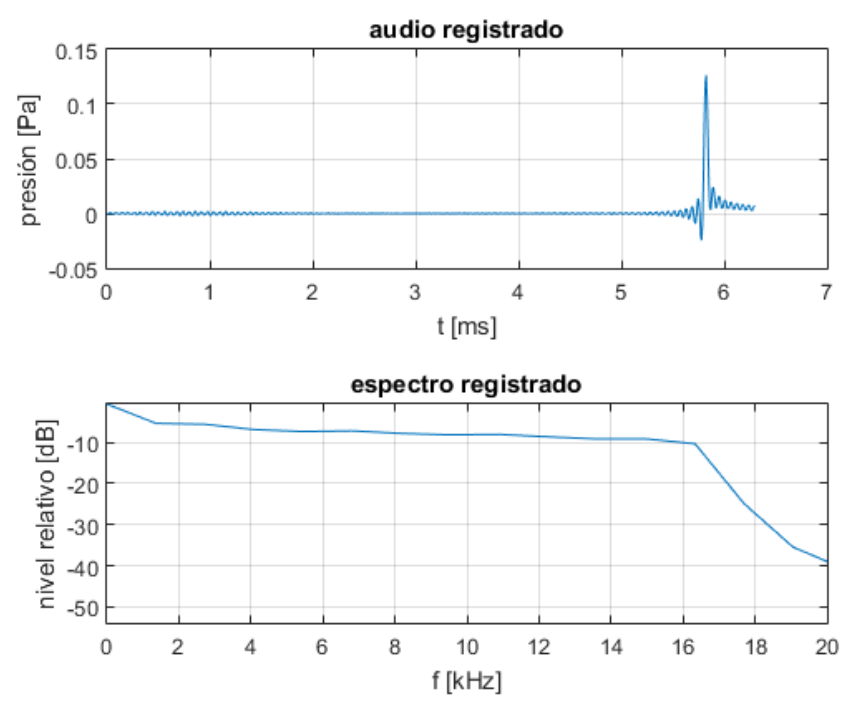

Fig. 5. Diagramas temporales de la señal registrada (respuesta al impulso) y su espectro.

\section{Convolución de la Respuesta al Impulso con el Audio}

Se convolucionó la respuesta al impulso con el audio original remuestreado a $114 \mathrm{kHz}$ (para respetar el $\Delta t$ de procesamiento de k-wave). El resultado fue nuevamente un audio claro y perfectamente inteligible, con un ligero incremento en frecuencias bajas. Tal como era de esperar, el audio recuperado posee un retardo de poco menos de 6 $\mathrm{ms}$ respecto del audio original, idéntico al de la simulación de propagación del audio completo.
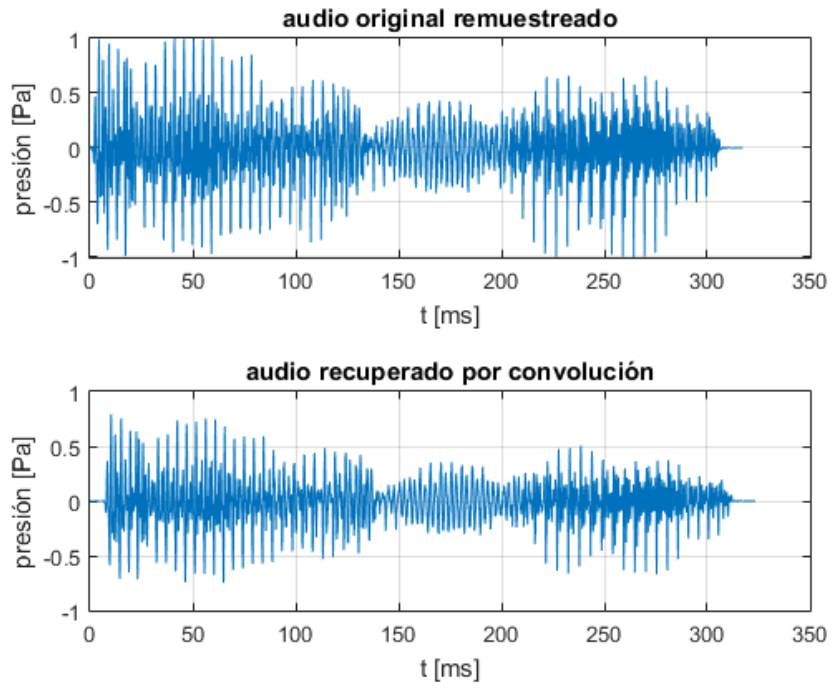

Fig. 6. Diagramas temporales del audio original emitido por la fuente y el audio registrado por el sensor.

\section{CONCLUSIONES Y TRABAJO Futuro}

Los resultados reportados resultan alentadores para continuar la exploración en profundidad de los métodos pseudoespectrales con miras a utilizarlos en auralizaciones. Las pruebas realizadas muestran que es posible obtener una respuesta al impluso en un solo ciclo de cómputo, y que el resultado de la propagación de una onda de audio completa coincide con la propagación de un impulso y su posterior convolución con el audio original, en forma consistente con lo que se espera de un sistema LTI. La función de transferencia en $3 \mathrm{D}$ resulta prácticamente plana en un rango de frecuencias adecuado para sus aplicaciones en audio. En las simulaciones en 2D se verifica un incremento en frecuencias bajas que podría ser compensado mediante filtros para obtener una respuesta plana.

Hemos realizado pruebas preliminares en ambientes de mayor tamaño incluyendo una variedad de configuraciones de superficies reflectantes con interesantes resultados. Estos trabajos necesitan ser verificados y sometidos a un análisis más cuidadoso antes de ser reportados.

Parte de nuestro equipo de investigación ha comenzado a explorar con diferentes materiales (densidad, velocidad de propagación, absorción), con la intención de repetir las experiencias de propagación de audio en situaciones más complejas. Resulta necesario continuar con una mayor exploración de los límites sobre los cambios que pueden ser introducidos en las características del medio sin que se pierda la posibilidad de simular la propagación de un impulso en un único proceso de cómputo.

\section{AGRADECIMIENTOS}

Los autores de este trabajo quieren agradecer muy especialmente al equipo conformado por Damián Fernández, Ianina Canalis, Ian Kuri y Nicolás Casais Dassie por sus aportes durante todo el proceso.

El presente trabajo es parte es parte de un proyecto de investigación que se encuentra en desarrollo en la Universidad Nacional de Lanús.

\section{REFERENCIAS}

[1] M. Vorländer, Auralization: fundamentals of acoustics, modelling, simulation, algorithms and acoustic virtual reality, Berlin, Alemania: Springer Science \& Business Media, 2008

[2] D. J. Meares, "The Use of Scale Models in the Acoustic Design of Studios," SMPTE J., vol. 87, no. 10, pp. 684-687, Oct. 1978

[3] G. M. Naylor, "ODEON-Another hybrid room acoustical model," Appl. Acoust., vol. 38, no. 2-4, pp. 131-143, 1993

[4] M. Schroeder, "Die statistischen Parameter der Frequenzkurven von großen Räumen," Acta Acust. united Ac., vol. 4, no. 5, pp. 594-600, Jan. 1954

[5] M. R. Schroeder, y K. H. Kuttruff, "On frequency response curves in rooms. Comparison of experimental, theoretical, and Monte Carlo results for the average frequency spacing between maxima," $J$. Acoust. Soc. Am., vol. 34, no. 1, pp. 76-80, 1962

[6] H. V. Fuchs, Applied Acoustics: Concepts, Absorbers, and Silencers for Acoustical Comfort and Noise Control: Alternative SolutionsInnovative Tools-Practical Examples, Berlin, Germany: Springer Science \& Business Media, 2013

[7] J. O. Smith, Physical audio signal processing: For virtual musical instruments and audio effects. San Francisco, CA, USA: W3K Publishing, 2018

[8] L. Chen, L. Liu, W. Zhao, y H. Chen, "2D acoustic design sensitivity analysis based on adjoint variable method using different types of boundary elements," Acoust. Aust., vol. 44, no. 2, pp. 343357, Jul. 2016

[9] M. Vorländer, "Computer simulations in room acoustics: Concepts and uncertainties," J. Acoust. Soc. Am., vol. 133, no. 3, pp. 12031213, Mar. 2013

[10] C. Canuto, M.Y. Hussaini, A. Quarteroni, y T. A. Zang, Spectral methods: Fundamentals in single domains, Berlin, Germany: Springer-Verlag, Berlin, 2006

[11] A. Solomonoff, y E. Turkel, "Global properties of pseudospectral methods," J. Comput. Phys., vol. 81, no. 2, pp. 239-276, Apr. 1989

[12] G. T. Huntington, y A. V. Rao, "A comparison between global and local orthogonal collocation methods for solving optimal control problems," en 2007 Amer. Contr. Conf. IEEE, pp.1950-1957.

[13] S. A. Orszag, "Comparison of pseudospectral and spectral approximation," Stud. Appl. Math., vol. 51, no. 3, pp. 253-259, 1972

[14] J. Virieux, H. Calandra, y R. E. Plessix, "A review of the spectral, pseudo-spectral, finite-difference and finite-element modelling 
techniques for geophysical imaging," Geophys. Prospect., vol. 59, no. 5, pp. 794-813, Sep. 2011

[15] K. Firouzi, B. Cox, B. Treeby, y N. Saffari, "A first-order k-space model for elastic wave propagation in heterogeneous media," $J$. Acoust. Soc. Am., vol. 129, no. 4, pp. 2611-2611, Apr. 2011

[16] Q. H. Liu, "The PSTD algorithm: A time - domain method combining the pseudospectral technique and perfectly matched layers," J. Acoust. Soc. Am., vol. 101, no. 5, pp. 3182, May. 1997

[17] Y. Jing, F. C. Meral, y G. T. Clement, "Time-reversal transcrania ultrasound beam focusing using a k-space method," Phys. Med. Biol., vol. 57 , no. 4, pp. 901, Jan. 2012

[18] B. Treeby, M. Tumen, y B. Cox, "Time domain simulation of harmonic ultrasound images and beam patterns in 3D using the kspace pseudospectral method," en Int. Conf. Medical Image Computing Computer-Assisted Intervention, Berlin, 2011, pp. 363370.

[19] J. Hargreaves, P. Kendrick, S. Von Hünerbein, "Simulating acoustic scattering from atmospheric temperature fluctuations using a $\mathrm{k}$-space method," J. Acoust. Soc. Am., vol. 135, no. 1, pp. 83-92, Jan. 2014
[20] F. Pind, M. S. Mejling, A. P. Engsig-Karup, C. H. Jeong, y J. Strømann-Andersen, "Room Acoustic Simulations using High-Order Spectral Element Methods," en Euronoise 2018, pp. 2085-2092.

[21] B. E. Treeby, y B. T. Cox, "k-Wave: MATLAB toolbox for the simulation and reconstruction of photoacoustic wave fields," $J$. Biomed. Opt., vol. 15, no. 2, Mar. 2010, Art. no. 021314.

[22] J. L. Robertson, B. T. Cox, y B. E. Treeby, "Quantifying numerical errors in the simulation of transcranial ultrasound using pseudospectral methods," en IEEE Int. Ultrasonics Symposium (IUS), 2014, pp. 2000-2003.

[23] M. Aretz, R. Nöthen, M. Vorländer, y D. Schröder, "Combined broadband impulse responses using FEM and hybrid ray-based methods," en Proc. EAA Symp. Auralization, Jun. 2009, pp. 201-206.

[24] Q. H. Liu, "The pseudospectral time-domain (PSTD) algorithm for acoustic waves in absorptive media," IEEE T. Ultrason. Ferr., vol. 45, no. 4, pp. 1044-1055, Jul. 1998

[25] G. Zhao, y Q. H. Liu, "The unconditionally stable pseudospectral time-domain (PSTD) method," IEEE Microw. Wirel. Co., vol. 13, no. 11, pp. 475-477, Nov. 2003 